\title{
EDITORIAL
}

\section{Treatment and hematopoietic SCT in aplastic anemia}

Bone Marrow Transplantation (2013) 48, 161; doi:10.1038/ bmt.2012.229; published online 19 November 2012

This edition of the journal Bone Marrow Transplantation is the dedicated to the diagnosis and treatment of aplastic anemia, a disease that has had an important role in developing SCT and maybe even modern hematology. This was borne out of a project to design algorithms for the diagnosis and treatment of this disease, comprising different clinical issues such as different age groups and disease severity, as well as strategies arising from the unavailability of a specific type of donor. Aplastic anemia is an orphan disease with an incidence of 1-2 patients per million inhabitants per year, and given the different paths in the algorithms this breaks down into even smaller distinct subentities. Without wishing to stress the common place of personalized medicine, these distinct situations require individualized attention. Often, the approach to patient care has to differ, be it while caring for the child or the older person being diagnosed with aplastic anemia, according to severity of the disease and response to initial treatment as well as depending on the availability or not of a matched donor. Given the difficulty to study rare diseases, we also designed these algorithms with the intention to standardize treatment and to record treatment outcome. Diagnostic procedures need to be standardized; congenital disease has to be ruled out in the young, hypoplastic myelodysplastic syndrome in the older patient and procedures are discussed in the chapter on the diagnosis of acquired aplastic anemia. Outcome of patients with aplastic anemia has greatly improved over the past decades and to a large part this is due to progress in supportive care, given that our currently used immunosuppressive and transplant regimens were developed in the 1970s. This progress includes treatment of infectious complications and transfusional support, among others. In younger patients, the primary decision in treatment is to choose between transplantation versus immunosuppression. We have learned recently through a randomized trial conducted at the National Institutes of Health that not all antithymocyte globulin regimens are equal. ${ }^{1}$ Standards for immunosuppression are to be found in the chapter on front line immunosuppressive treatment as well as current standards on upfront BMT with a sibling donor. Older patients with a donor are recommended to be treated with immunosuppression, but patients failing this treatment are candidates for BMT using a reduced intensity regimen containing fludarabine. Patients without a sibling donor failing immunosuppressive treatment may have an unrelated donor search initiated and may undergo marrow transplantation from an unrelated donor. Transplant rates in Europe for aplastic anemia continue to increase ${ }^{2}$ and this is mostly due to the increased use of unrelated donors following publications showing that outcomes with unrelated donors were similar to outcomes with sibling donors. The average age of patients with aplastic anemia is $\sim 16$ years, that is, half the patients are children, but the disease can occur in all age groups. There is a specific chapter on the management of aplastic anemia in children and also on the management of aplastic anemia in the older adults. The issue is rounded up by particular chapters on second- and third-line treatment options, including cord blood transplantation, transplantation from mismatched family donors (that is, haploidentical donors) and experimental immunosuppressive regimens including Campath. These specific protocols are detailed here to incite treating physicians to manage these patients according to common treatment protocols and to report outcomes in order for us to progress toward improved outcomes for these patients. All these chapters have been written by members of the aplastic anemia working party of the European Group for Blood and Marrow Transplantation and the algorithms are the fruits of many discussions held during our biannual group meetings. We hope to provide a platform for the reader that may help him in all clinical situations that can be encountered with these patients. For this reason, the working title of this project had been 'the complete treatment algorithm'.

\author{
JR Passweg ${ }^{1}$ and M Aljurf ${ }^{2}$ \\ ${ }^{1}$ Klinik Hämatologie, Bereich Innere Medizin, \\ Universitätsspital Basel Basel, Switzerland and \\ ${ }^{2}$ King Faisal Specialist Hospital and Research Centre, \\ Riyadh, Saudi Arabia \\ E-mail: jpassweg@uhbs.ch
}

\section{REFERENCES}

1 Scheinberg P, Nunez O, Weinstein B, Scheinberg P, Biancotto A, Wu CO et al. Horse versus rabbit antithymocyte globulin in acquired aplastic anemia. $N$ Engl J Med 2011; 365: 430-438.

2 Passweg JR, Baldomero H, Gratwohl A, Bregni M, Cesaro S, Dreger $\mathrm{P}$ et al. and for the European Group for Blood and Marrow Transplantation (EBMT). The EBMT activity survey: 1990-2010. Bone Marrow Transplant 2012; 47: 906-923. 